\title{
The Study of Information Integration in EIS Based On Grid
}

\author{
Renjing Liu and Jing Tian \\ School of management, Xi' an Jiaotong University, Shaanxi, \\ 710049,renjingl@163.com
}

\begin{abstract}
This paper first illuminate the literature survey of the EIS and Grid research, and then introduces the Grid conception. Then the paper establishes a system topology Structure description. In the topology structure used the host and subsidiary node conception, based on the topology structure, the EIS information integration frame structure based on Grid is established. The paper gives a detailed description of the structure. Then the paper conceived the safety EIS access mode. At last, the paper sums up the advantages of the using grid technology into EIS information integration.
\end{abstract}

\section{Introduction}

\subsection{The research background}

With the popularization of the computer and the development of network technology, many enterprises introduced or have voluntarily developed the enterprise information system (EIS) to improve their operational activity efficiency nowadays, but during the process of application, the information integration and the data access form standard disunity makes information unable to share and collectively manage, and then formed the so-called " information isolated island ". In order to dispel this phenomenon, the application of the Grid technology with the purpose of resources integration and cooperation share to construct the enterprise information system has become inevitable trend in coordination.

Traditional information data integration management use the way like this: pick up the needed information from the subsystem or terminal equipment to center equipment for use and access. This process cause many problems. The Grid

Please use the following format when citing this chapter. 
technology comes into being has made the problems solve more easily and also obviously reduced the cost and risk, and then the user will also obtain better services.

\subsection{Influence of Grid Technology on EIS}

The grid caused all kinds of information and application procedure that distribute in the world carry on the seamless fusion and the communication, thus forms the brandnew commercial opportunity for an enterprise.

Use the Grid in EIS can help us avoid the Information isolated island; realize the integration of the logistics, financing flow, information flow and the task flow.

The Grid solve the data isomerism, enable the EIS unified manage the information and make the process of information share and integration easier.

\section{Grid Technology Introduction}

If there existed a technology which connects large amounts of computers located at different places through net, it can make a visual super-computer by share the idle processing power, which would efficiently make up for the scarce of resources, saving the money being invested, sharing resources as well. This technology is namely Grid Technology.

Grid is an infrastructure which provides comprehensive computer resource and service with sharing as its core. It can realize the all-round sharing of computer, storage, data, information, knowledge, experts etc. The core conception of Grid is an old saying "Grid is computer".

The essence of Grid is not its size but by the sufficient use of the software and hardware existed in the net support the calculation, data, storage, information share etc. in the WAN. By using Grid, we can avoid the Information isolated island; obtain high performance by the low cost.

Grid is firstly the resources integration extending to the management domain and then the solution technology based on these resources.

The characteristic of the Grid is: 1) Low cost; 2) Resources strong distribution; 3) Isomerism; 4) No protocol standard generally accepted; and 5) Autonomy, Dynamic, and Extension Resource

The Grid tries to realize the connection of all the resource in the net, make people use the net as comfortable as use the electricity and don't need to consider where it comes from. It is called Think \& Obtain. 


\section{EIS Information Integration Frame Structure Based On Grid}

\subsection{System Topology Structure Description}

Many Enterprises use the data concentrates to pick up the needed information from the terminal equipment to the center equipment. This process cause many problems, such as the center equipment burden is overweight, or ask for high performance and high cost, real-time renews is difficult and so on. Here use the topology structure to set some nodes, including host nodes and subsidiary nodes, the host nodes use network structure and one host node can attach some subsidiary node. The disperser controls are used here. Fig. 1 shows the topology structure.

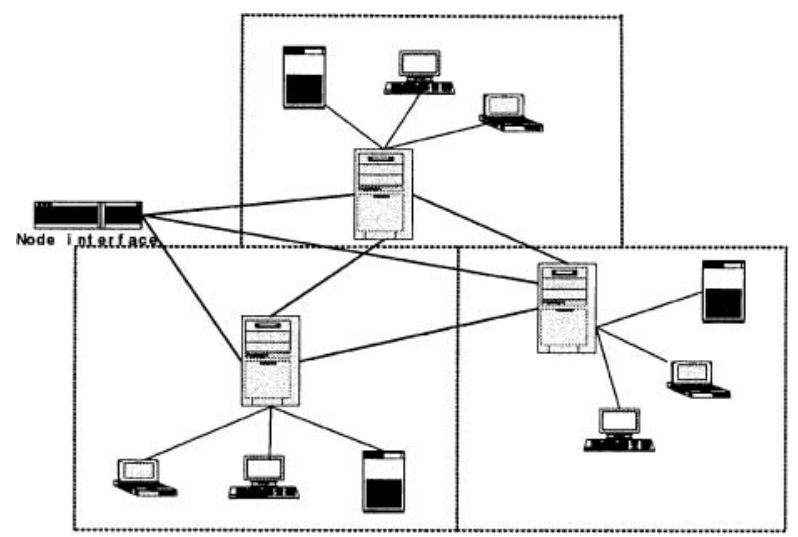

Fig. 1 System Topology Structure

Because the use of the disperser control, even the local network breakdown cannot affect the entire system. The system has very high reliability. Each node established a straight link, and the information flow is the shortest. The node construction emphasize on coordinated and mutually operation. All the nodes provide resource for sharing and monitor, optimize these resources through the host node. The system uses service interface to extend nodes.

\subsection{EIS Information Integration Frame Structure Description}

In EIS the information integration goal is not establish an entity database, but is integrate the data dynamic information into unification virtual view. The original data in local is still used. 
The EIS system structure is divided into 3 levels. It shows the mapping that from the domain application layer to the infrastructure layer through the grid service and the grid middleware level. Consider current EIS platform information integration demand and grid technology development condition, the author provided the entire EIS information integration frame structure, based on three-level systems structures, Fig. 2 shows the main level and the function configuration.

We can see that the three main parts of the structure is: Infrastructure, Grid intermediate level, and the domain application. The infrastructure is the foundation, the Grid intermediate is the core and the domain application is the purpose.

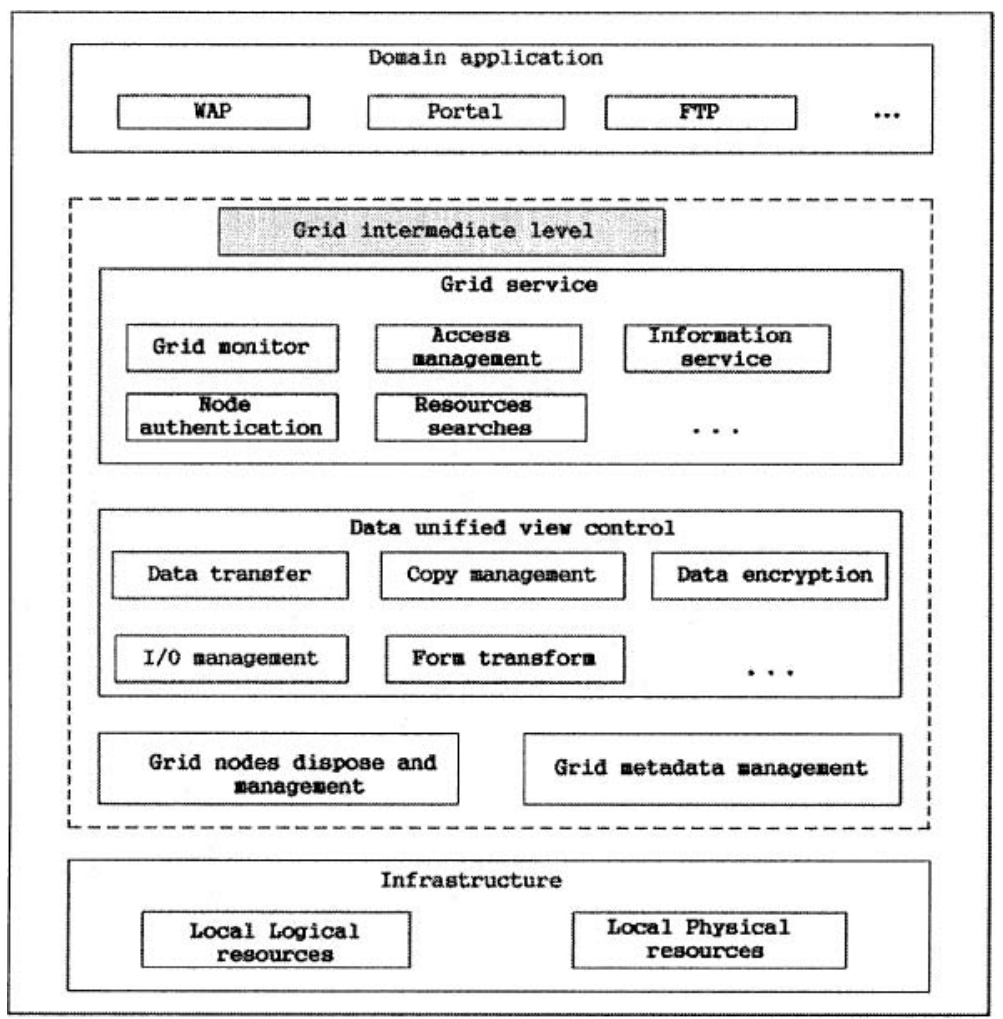

Fig. 2 EIS Information Integration Frame Structure

\section{-Infrastructure}

Provides the basic network strut environment to be qualified needs the local logic and the physical resources which shares.

\section{-Grid intermediate level}

This level is the core of the whole EIS, which transform the data form, manage the data and provide public service. This part can be divided into three parts: 
(1) Grid nodes dispose and management\& Grid metadata management Dispose the nodes, manage and monitor the nodes. Pick up the metainformation from the nodes and doing management and correlative operation.

(2) Data unified view control This is the main part of the Grid intermediate level. The view control provides data pick-up, form transformation, information transfers, and so on.

(3) Grid service Provide basic grid service, such as Grid monitor, node authentication, access management and so on.

\section{-Domain application}

For different application domain provide different application, and for different application there are different visit forms. Provide the access interface to other users.

\subsection{EIS integrated Information Access Mode}

The enterprise information data distributes in the different nodes, the dynamic changed data provides different service to different users. Here based on the system topology structure and information integration frame, we establish the EIS integrated information access mode, Fig. 3 shows the mode.

The EIS access mode manifests the characteristic as followed:

\section{-Security}

Local login make sure the user is legal and the node authentication make the user legally login the neighborhood nodes.

\section{-Transparency}

When login, the users face a unified data view. EIS hides the integration detail and then the user access the information data on other nodes as if use the local information data.

\section{-Good service}

The topology structure made the whole system can store more data than before, and because of the host nodes buffer, the user can obtain high handle speed.

\section{-Openness}

The system can accommodate and handle all kinds of different form data.

Fig. 3 EIS Integrated Information Access Mode

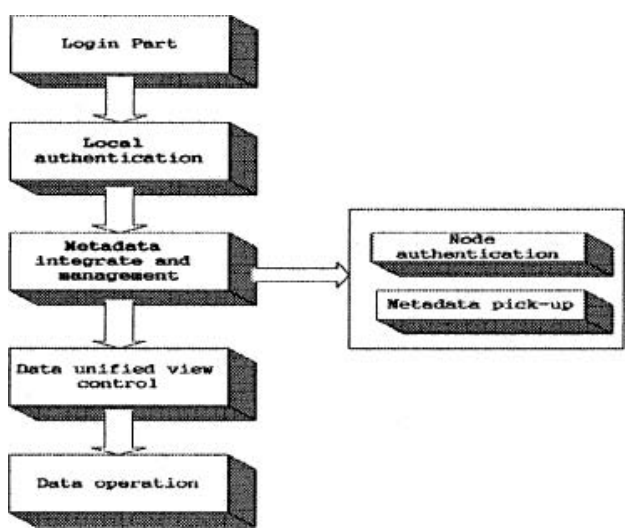




\section{Conclusions}

The enterprise data is the most important numeral resources of EIS, by establishing the EIS information integration frame structure; we realized the data sharing in EIS and simplified the system operation. The structure aimed to implement the whole system visit and seamless integration; enhance the local data control and maintenance; use the original data and resources to the great extent. The safe access mode is applied, and then reduced the information data transfer. The design shows the advantages of using grid technology into the EIS construction. Because of the time and knowledge limits, the application detail needs further research.

\section{Acknowledgements}

The authors would like to thank Professor Lida $\mathrm{Xu}$ for his insightful and helpful comments. We are grateful for the funding from Bureau of Science and Technology in Shaanxi Province (Grant No. 2004KR59), and "985" two term "07" base in Xian Jiaotong University.

\section{References}

1. G. Xiaoling, Grid Computing Technology (Beijing Post Press, Beijing, 2005).

2. X. Jun, Grid Computing and E-Learning Grid: Architecture, Key Technologies and Applications (Science Press, Beijing, 2005).

3. Z. Chunxia and X. Huaping, Enterprise Data Integration and Interoperability Based on Grid, Computer and Digital Engineering 33(11), 49-52 (2005).

4. I. Foster, What is the Grid: A Three Point Checklist, GRID Today, (2002).

5. X. Jun, P. Nan and W. Ning, The Inference and Revelation of the Network Technology on Management of Enterprise, Science Progress and Countermeasure 3, 87-88, (2005). 